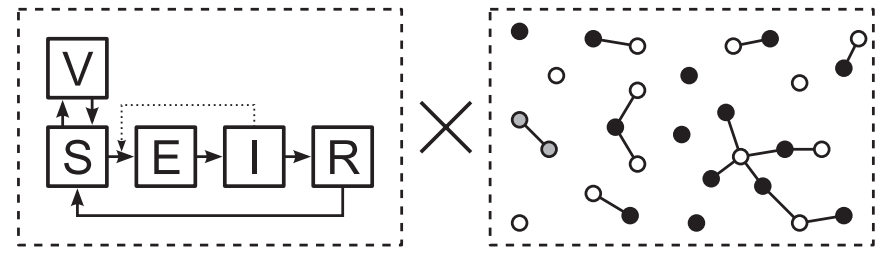

Abstract P1-S4.19 Figure 1 Epidemiology on networks.

\section{P1-S4.20 MATHEMATICAL MODELLING OF HIV TRANSMISSION AND CONTROL AMONG MEN WHO HAVE SEX WITH MEN: A REVIEW OF 25 YEARS OF LITERATURE}

doi:10.1136/sextrans-2011-050108.164

${ }^{1} \mathrm{~N}$ Punyacharoensin, ${ }^{1}$ W J Edmunds, ${ }^{2} \mathrm{D}$ De Angelis, ${ }^{1} \mathrm{R}$ Guy White. ${ }^{1}$ London School of Hygiene \& Tropical Medicine, London, UK; ${ }^{2}$ Heath Protection Agency and MRC Biostatistics Unit, UK

Background For a quarter of century, mathematical models have been used to study the spread and control of HIV among men who have sex with men (MSM). We reviewed this literature to summarise the methodologies used, key model developments, and recommended strategies for HIV control among MSM.

Methods Review of the literature on dynamic compartmental models of HIV transmission among MSM was conducted. MEDLINE/EMBASE were searched from earliest date to end 2010.

Results Of 742 studies identified, 127 studies met the inclusion criteria and were selected for review. Most studies employed deterministic methods (80\%), and potentially as a result of this, as the complexity of models increased over time with respect to antiretroviral therapy (ART), there was a marked decline in the complexity of models with respect to sexual activity. Only a small proportion of models were fitted to data (22\%) and even fewer were validated $(17 \%)$, somewhat reducing confidence in the findings from these studies. That said, a number of common findings emerged, including (1) the importance of assumed changes in infectivity and sexual contact rates on the impact of ART on HIV incidence, and that this led to follow-up empirical studies to gather these data, and (2) the recommendation that multiple strategies would be required for effective HIV control among MSM.

Conclusions Mathematical models have been useful in indentifying key empirical studies and for showing that multiple prevention strategies would be required for effective control of HIV epidemics in MSM. The lack of model fitting and validation emphasise that this area should be targeted for developments in the future. An improved methodology for parameter estimation will help generate predictions that more fully express uncertainty, allowing more informed public heath decision making.

\section{P1-S4.21 CALIBRATION OF AN INDIVIDUAL-BASED MODEL OF STI TRANSMISSION IN UGANDA: A NOVEL ABC-BAYESIAN EMULATION HYBRID APPROACH}

doi:10.1136/sextrans-2011-050108.165

H Johnson, W John Edmunds, R White. London School of Hygiene and Tropical Medicine, London, UK

Introduction The complexity of dynamic epidemic systems has led to the use of large-scale stochastic models for prediction purposes. However, methods for robustly calibrating and analysing these models can be prohibitively inefficient. We propose an algorithm for fitting complex models that incorporates elements of both Approximate Bayesian Computation $(\mathrm{ABC})$ and Bayesian Emulation. $\mathrm{ABC}$ enables inference about model parameters without needing to calculate a likelihood function, by generating approx- imations from repeated model runs. However, each model run might take hours. Emulation methods are being developed in the fields of cosmology and meteorological modelling. The complex model function is summarised as an "emulator": a stochastic function that represents global behaviour of the function as a linear regression model and local deviations from this behaviour as Gaussian processes. The emulator acts as a cheap proxy for the complex model, allowing both calibration and sensitivity analysis to be conducted in a fraction of the time.

Methods We report the initial application of an emulation-based calibration algorithm to an individual-based stochastic model of STI transmission in Uganda. Starting with uninformative priors for 19 behavioural and biological input parameters, we "trained" an emulator with 200 sampled parameter sets and their corresponding model output (point estimates of HIV prevalence). Sampling a further 10000 parameter sets from the priors, we used the emulator to make output predictions over a large area of input parameter space. Weighting each parameter set by goodness of fit to observed data, we identified promising areas of parameter space for complex model evaluation. A more accurate emulator was then trained, incorporating this additional complex model output. The process was repeated as in sequential $A B C$ methods.

Results The use of emulators allowed evaluation of large areas of parameter space due to increased computational efficiency. Processing time for one prevalence point estimate was reduced from over $15 \mathrm{~min}$ on an HPC cluster to less than $0.1 \mathrm{~s}$ on a PC. Even the first two waves of such an algorithm provided helpful insight into the most influential parameters

Conclusions The development of an ABC-Bayesian Emulation hybrid approach to complex model calibration is promising. Emulators offer large advantages in computational efficiency. However, further research is needed regarding weighting, tolerance levels and covariance.

\section{P1-S4.22 IMPACT OF A HYPOTHETICAL CHLAMYDIA VACCINE ON POPULATION PREVALENCE: A MATHEMATICAL MODELLING STUDY}

doi:10.1136/sextrans-2011-050108.166

${ }^{1} \mathrm{~J}$ Heijne, ${ }^{1} \mathrm{C}$ Althaus, ${ }^{1} \mathrm{~S}$ Herzog, ${ }^{1} \mathrm{~N}$ Low. ${ }^{1}$ University of Bern, Bern, Switzerland

Background It has not yet been possible to develop a Chlamydia trachomatis (chlamydia) vaccine but research to achieve this continues. It is therefore important to evaluate the potential impact of different chlamydia vaccine strategies on population prevalence. The optimal strategy might differ depending on whether a vaccine prevents chlamydia infection, or prevents ascending infection to reduce the risk of long term complications. Here, we used a mathematical model to focus on vaccination strategies that aim to reduce population prevalence.

Methods We developed a deterministic pair model of heterosexuals aged 15-39 years that incorporates the formation and dissolution of sexual partnerships. We used sexual behavioural data from UK population-based studies to inform the model. The model has a baseline chlamydia prevalence of $3 \%$. In all strategies examined, vaccination was introduced before sexual debut. We investigated the impact on population prevalence of different types of vaccine coverage, vaccine efficacy and duration of protection. We also assumed different types of protection after vaccination. We started with full protection, but since animal models have shown that sterilising immunity is difficult to achieve we also investigated partial protection of the vaccine by assuming a decreased susceptibility to infection after vaccination or, when infected, a shorter duration of infection or a reduced transmission probability. 